\title{
The Minority Game with interactions
}

\author{
Inés Caridi ${ }^{1}$, Horacio Ceva ${ }^{2}$ \\ Departamento de Física, Comisión Nacional de Energía Atómica, Avda. del \\ Libertador 8250, 1429 Buenos Aires, Argentina
}

\begin{abstract}
We partially modify the rules of the Minority Game (MG) by introducing some degree of local information in the game, which is only available for some agents, called the interacting agents. Our work shows that, for small values of the new parameter of the model (the fraction of interacting agents), there is an improvement of the use of the resources with respect to the MG, while as this number grows the response of the system changes, and ends up behaving worst than the usual MG.
\end{abstract}

Key words: Minority game; interacting agents; entropy rate

\section{Introduction}

The minority game (MG) is an adaptive game suitable to study competitive systems whose available resources are finite [1]-[3]. In spite of the simplicity of the rules of the game, it presents a very rich behavior and interesting properties.

In this game there is a number of players (agents) that must choose, independently, one of two alternatives at each time step, splitting into two groups. The winners are those who stay at the minority side in that step. As we explain in the next section, there are three main ingredients in the game: (i) a public history, with the information of the $m$ previous minority sides; (ii) some prescriptions (strategies) to play with, and (iii) a reward system, whereby points are given to winning agents and strategies. These ingredients induce an emergent coordination whereby the system can be more efficient (in a sense explained below) than a random game. In fact, this is the main reason for the interest shown by many people on the behavior of the model.

1 email: caridi@cnea.gov.ar

2 email: ceva@cnea.gov.ar 
A recent line of research introduces modifications into the $\mathrm{MG}$, designed to approach the behavior of the players to that of the agents in a real market. Agents in an economy take decisions based on heterogeneous external information available at each time, plus internal information, including their personal preferences. The heterogeneous external information can be either global, such as an aggregate variable, or local. Some of those investigations study the effect of changes in the external information available in the MG. The work of M. Paczuski et al. [4] develops a version of Kauffman's model with certain rules of the MG. Other works introduce versions of the MG with local information. Between these, that of Kalinowski et al. [5] places the agents on a circle; every agent gets the previous decisions of her $(m-1)$ neighbors (half to her right, half to her left) as an input information; together with her own decision, she builds up a history of $m$ bits. In this form there is a local history for each player. The rest of the rules are as in the usual MG. S. Moelbert et al. [6] recently introduced a local $M G$, where they use a local determination of the minority and the corresponding local histories.

In this work some kind of local information will be made available to a fraction $p$ of the $N$ agents $(0 \leq p \leq 1)$; we will refer to them as the interacting agents. As we will see, they will be able to change their original decisions, in order to be in the minority of their neighborhoods.

In the next section we describe the model, and present the results found; in section 3 some conclusions are drawn.

\section{The MG with interactions}

\subsection{The model}

In the MG model there are $N$ agents, each one with $s$ strategies distributed at random (with reposition) at the beginning of the game. The strategies assign one output ( 0 or 1 ) for every one of the $2^{m}$ possible inputs (i.e., histories). As a result of their choices, at each step of the game the players split into two groups of size $N_{0}$ and $N_{1}$, such that $N_{0}+N_{1}=N$. Every winner agent gets a point after each step of the game. Besides, out of all the strategies playing in the game, those that correctly predict the minority side of the time step $t$, obtain a reward, called a virtual point. The agents use the virtual points to establish the performance of her strategies and to choose, at each step of the game, the more successful one to play with.

The main variable considered in this model is $\sigma$, the standard deviation of the 
difference $\left(N_{1}-N_{0}\right)$

$$
\sigma^{2}=\frac{1}{T} \sum_{t=1}^{T}\left(N_{1}-N_{0}\right)^{2}
$$

where $T$ is the number of time steps of the game. This variable measures the form in which the resources of all the players are used. When $\left|N_{1}-N_{0}\right|$ is small, the minority group is bigger, and the agents, as a whole, receive more points. Hence, smaller values of $\sigma$ indicate a better use of the resources by the population. As mentioned above, the interest on this model is mainly due to the fact that for certain values of the variables $m, s$, and $N$, the standard deviation turns out to be smaller than in the case of a random game. The characterization of this collective behavior generated many studies of the MG, with a variety of techniques: numerical simulations [1], [7]; mean-field approximations [8]; equivalence with spin-glass models [9]; thermal treatments [10]; etc.

To introduce local information, we need to arrange the agents in such a form that it makes sense to talk about the neighborhood of every one of them. Hence, we distribute all the players on a square lattice with periodic boundary conditions. The positions of the $p N$ interacting agents on the lattice are chosen randomly, at the beginning of the game. At each time step the agents follow the usual rules of the MG, but the interacting agents (I.A.) are given the extra opportunity to modify their bets, after knowing what their nearest neighbors I.A. will do in the same step. As they were distributed randomly, every one of them can have between zero and four I.A. as nearest neighbors. This agent will try to be in the minority of the group formed by her $I$.A. nearest neighbors, plus herself. Hence, if more than half of her neighbors choose one side, then the I.A. will chose the other, regardless of what her best strategy says; but if half of the neighbors choose each side, then the $I$.A. will decide in agreement with the prescription of her best strategy, i.e. the one with more virtual points. With this rule, she can eventually choose one option that none of her strategies prescribe for a given history. Once all the agents have made their choice, they make their moves simultaneously. Finally, one obtains the minority as usual, by considering the actions of all the players. As far as the players are concerned, points are distributed with the same rule than in the MG, but whenever a player wins because she plays against the majority of her local neighborhood, then no points are added to her strategies.

The fact that the $I$. A. positions are chosen randomly raises the question of the relevance of the actual number of links between interacting agents, $\ell$, for every specific realization (i.e. for every sample), for a fixed value $p N$. We considered this factor, and found that in this case it only has minor effects [11]. 


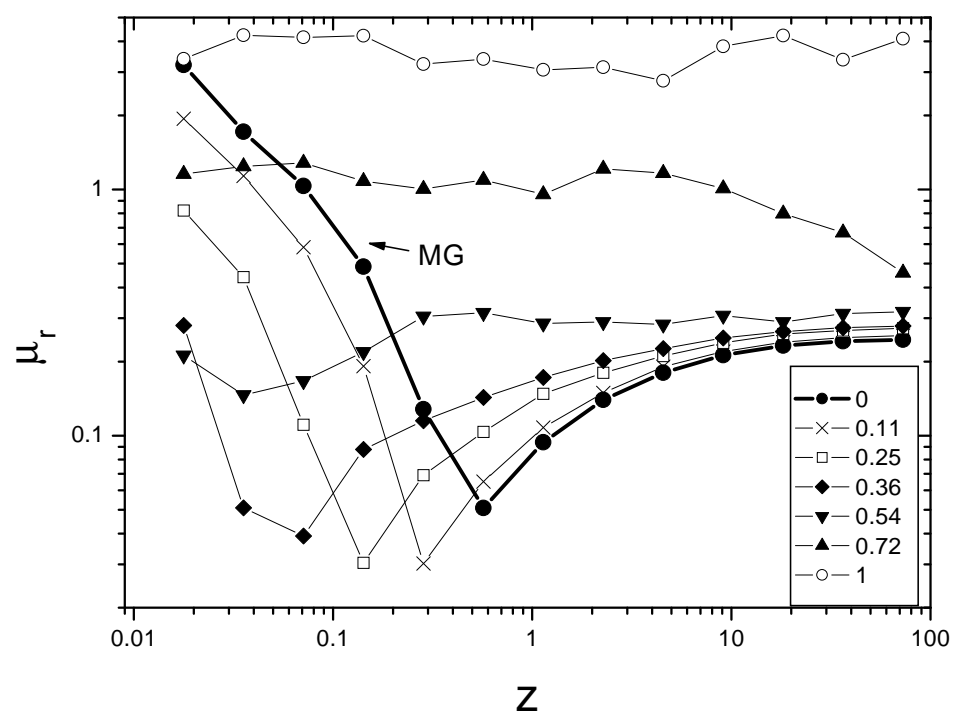

Fig. 1. Reduced variance $\mu_{r}$ as a function of $z$, for $N=225$ agents, and several values of $p$, indicated in the inset. Out of a total of $10^{5}$ time steps, data was collected in the last $9 \times 10^{4}$ steps.

\subsection{Results}

We have made extensive numerical simulations, finding $\sigma^{2} / N$ as a function of both $p$ and $2^{m} / N$. In the following we will refer to $\mu_{r} \equiv \sigma^{2} / N$ as the reduced variance, and use $z \equiv 2^{m} / N$, for short. As is well known [7], the graph of $\mu_{r} v s$ $z$ displays two different informational behaviors: an efficient phase for $z<z_{c}$, and an inefficient phase for bigger values of $z$. Qualitatively, $z_{c}$ is the greatest value of $z$ for which the system is dominated by a period-two, maladaptive dynamics that is explained below.

In Fig. 1 we can see the ensuing changes in the reduced variance, as $p$ increases from $p=0$, i.e. the $\mathrm{MG}$ with no interacting agents, to $p=1$, when all the agents are interacting. For small values of $p$ the function $\mu_{r}(z)$ has a shape similar to that of the MG case, but there is a shift to the left of its minimum, implying an improved efficiency for small values of $m$. Moreover, the minimum $\mu_{\text {min }}$ is smaller than the corresponding MG value. As $p$ increases, the shape of the curve changes, and for $p=1$ the reduced variance is roughly constant, and the efficiency is clearly worst than in the MG case.

In the following we will restrict ourselves to consider the first, informationally efficient region. We now will relate this behavior with the crowd - anticrowd picture [7], [12].

The minority game has, for $m=2$, a remarkable period-two dynamics (PTD), 

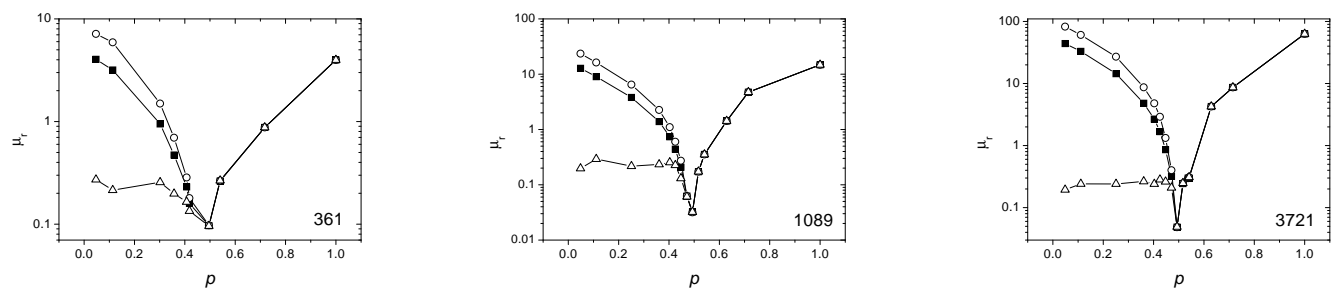

Fig. 2. Reduced variance $\mu_{r}$ as a function of $p$, for $m=2$. The different values of the reduced variance are calculated using $\sigma_{e}$ (empty circles), the whole dispersion $\sigma$ (filled squares), and $\sigma_{o}$ (empty triangles).

whereby during the odd appearances of a given history, the resulting minority is random, but then in the following, even appearance, the minority is just the opposite to the previous one. Moreover, if we distinguish even and odd occurrences of the histories, i.e. we calculate the standard deviation $\sigma_{e}\left(\sigma_{o}\right)$ for the time steps in which the histories occur an even (odd) number of times, it turns out that $\sigma_{o}$ is of the order of $\sqrt{N} / 4$, while $\sigma_{e} \gg \sigma_{o}$. If $m=2$, this is true for almost all the steps of the game; as $m$ increases, this rule is true only in a (decreasing) number of steps, and when $z \approx 10$ or greater, the opposite happens: the 'even' minority tends to be the same than the previous, 'odd' one. This was first observed and explained by Manuca et.al. [7], as due to the formation of crowds (see also [12], [13]). In a few words, this happens as follows: during the first (odd) occurrence of a history, the strategies of the players have the same amount of virtual points, and therefore they will pick side randomly, so that $N_{0} \approx N_{1}$; thereafter, the winner strategies will get a point. During the second (even) appearance of the same history, agents having those strategies will bias the outcome, choosing the same side as before. Hence, the minority will be just the opposite than the previous one; on the other hand, $N_{0}$ and $N_{1}$ will be very different, so that $\sigma_{e} \gg \sigma_{o}$. Now the strategies receiving virtual points are those complementary to the first set; in this form all strategies have roughly the same amount of points, closing a 'cycle'.

We begin by studying the effect of the interactions on the $m=2$ case; as we will see, for small values of $p$ the system still follows the PTD, but as $p$ increases the period-two regime will disappear.

Figure 2 shows data for $\mu_{r}$ as a function of $p$, for $N=361,1089$ and 3721 (these are just three representative examples of all the cases we have considered). We discarded the first $10^{4}$ time steps for $N=361$, and $510^{4}$ for bigger values of $N$, and collected data along the next $10^{5}$ time steps. All the results shown are averages over 32 independent realizations.

Each set has three curves, $\mu_{r}^{o}(p), \mu_{r}^{e}(p)$ and $\mu_{r}(p)$,corresponding to the odd, even and total number of appearances of the histories, respectively. It is easy to distinguish three regions: $(i)$ zone 1 , where $\mu_{r}^{o}(p) \approx 0.25 \ll \mu_{r}^{e}(p)$. This is the PTD region, as can be verified by the simple observation of the record 
of minorities. As it is known, 0.25 is the expected value for a random set; (ii) zone 2 , an intermediate region where $\mu_{r}^{o}(p)$ is no longer that of a random process, but still $\mu_{r}^{o}(p)<\mu_{r}^{e}(p)$; and finally, (iii) zone 3 , a region where the even and odd curves coincide and, therefore, there is no PTD present. Notice that the width of the intermediate zone shrinks as $N$ increases. For $m=2$ and $N=3721$, the 3 rd zone starts at $p^{*} \simeq 0.5$ (this value has only a moderate size effect).

The behavior for small values of $p$ can be understood as a simple extension of the behavior of the MG: there is only a small number of I.A., so that they can reduce somewhat the value of $\sigma$, but can not change the general outcome of the game. It is also possible to explain in simple terms the behavior for $p=1$. In this case, all the players are interacting, hence each one has a neighborhood of $4 I$.A. Let us assume, for the sake of concreteness, that the minority outcome for an odd appearance of a given history is $\mathcal{M}=1$; we could expect that in the next (even) appearance of the same history, the majority of the players will pick the same side, as the PTD indicates. With the new rules, however, there is an extra opportunity for all the players. Now, most of the agents will notice that their neighbors will chose ' 1 ', and therefore each one in this condition will, in turn, change her bet. As a result, the majority will chose ' 0 ', and the minority will again be ' 1 '; virtual points are given to the same group of strategies than before, in agreement with the previous crowd effect: each history will be followed always by the same answer. One consequence of this behavior, is that the system will fall into a cyclic evolution, with a period equal to 4 (the number of histories), or smaller.

Notice that, in general, $p^{*}$ is a function of $z, p^{*}(z)$. In the region between $p^{*}$ and $p=1$, the system behaves qualitatively in the same form. We found it appropriate to analyze the sequence of minorities of the game, $\left\{\mathcal{M}_{i}\right\}$, determining the entropy rate [14] of the set. The entropy rate of $n$ random variables $\left\{X_{i}\right\}$ measures how does the entropy of the sequence growth with $n$. If the stochastic process is a stationary Markov chain, the entropy rate $H_{T}(X)$ can be written as follows:

$$
H_{T}(X)=-\sum_{i, j} \lambda_{i} P_{i j} \log _{2} P_{i j}
$$

In this equation, $\lambda=\left(\lambda_{1}, \lambda_{2}, \ldots\right)$ is a vector whose components are the stationary probabilities of $X_{1}, X_{2}$, etc. $P$ is a probability transition matrix, whose elements measure the probability of the transition between the states $i$ and $j$, i.e. $P_{i j}=\operatorname{prob}\left\{X_{n+1}=j \mid X_{n}=i\right\}$. The reason to consider here this magnitude is the following: if the sequence of $m$-histories resulting from $\left\{\mathcal{M}_{i}\right\}$ has a cyclic behavior, the corresponding entropy rate is zero, because in this case $P_{i j}$ is either 0 or 1 . In other words, $H_{T}$ is an efficient detector of cyclic behavior. From a physical point of view, once one knows in what kind of cycle is the 


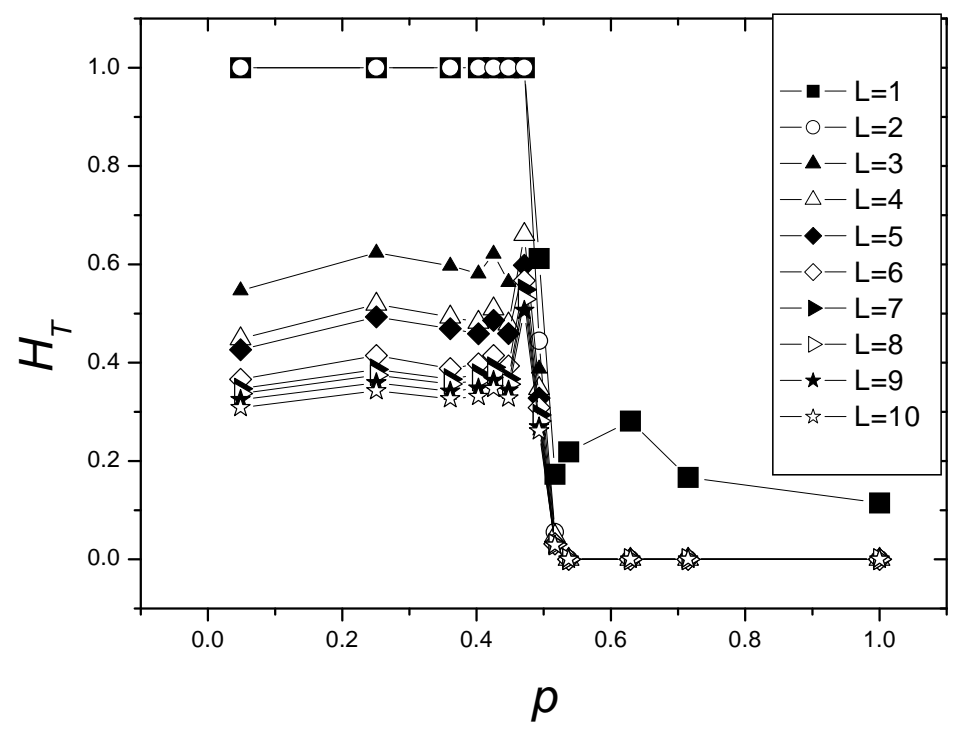

Fig. 3. Entropy rate $H_{T}$ as a function of $p$, for $N=3721$ agents and $m=2$. The symbols representing the different word sizes are shown in the inset. In this case $p^{*} \simeq 0.5$. Notice that for $p<p^{*}$, and $L \leq m$ the entropy rate is $H_{T}=1$, while for $p>p^{*}$ and $L \geq m$ the entropy rate is $H_{T}=0$.

system, there is no uncertainty about the next time step; that is the reason why the entropy rate vanishes.

The set $\left\{\mathcal{M}_{i}\right\}$ can be studied by forming $M-L+1$ 'words' of length $L$ out of the sequence of $M$ minority sides; two successive words will share $L-1$ values, or bits; thus for example if we want to look at words of length $L=3$, and the sequence of minorities is represented by ...abcde... (where each letter can take the values 0,1 ) one word will be 'abc' and the following 'bcd', etc. [15]. We determined $H_{T}$ for $m=2,5$ and 8 , and several values of $p$ in the interval $\{0,1\}$, using word sizes $L=1$ to 10 (see Fig.3). For $p \gtrsim p^{*}$ we found that $H_{T} \approx 0$, indicating the presence of a cyclic evolution. In fact, a close inspection of the series of minority sides shows that for $p$ in the interval $\left\{p^{*}, 1\right\}$, the system falls in a cyclic motion for a rather long number of time steps, until it suddenly switches to another cycle, and so on. The contribution to the entropy rate from the transition time steps between two different cycles is very small, but noticeable. As $p$ approaches $p=1$, the change of cycle becomes more and more uncommon.

Two other aspects of the results shown in Fig.3 deserve to be emphasized. In the first place, it is rather clear that the general behavior of the entropy rate follows that of the reduced variance, in the sense that both show two different 'phases', for the same values of $p$ : one 'phase' for $p \leq p^{*}$, and a different one for $p>p^{*}$. On the other hand, the entropy rate is roughly constant in the 

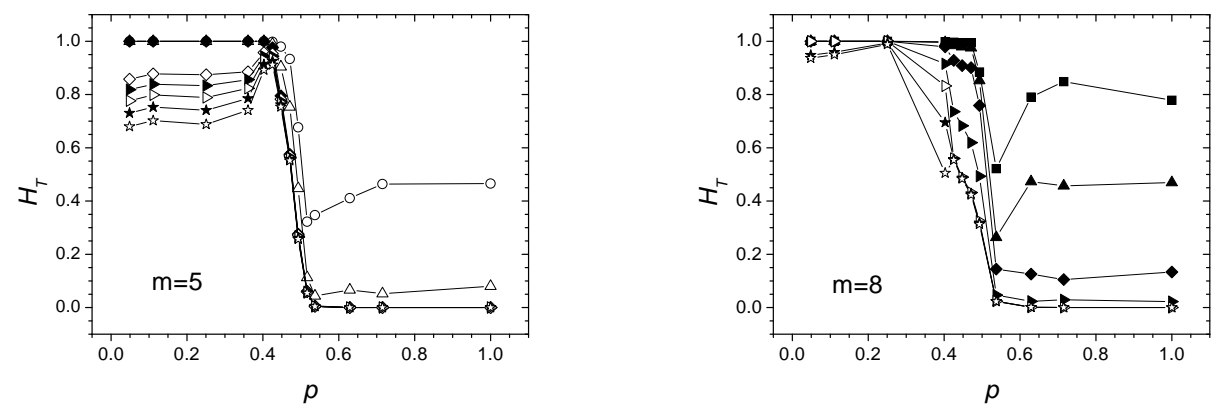

Fig. 4. Entropy rate as a function of $p$, for $m=5$ and 8 . We use the same symbols as in Fig.3. For clarity we only show results for selected values of $L$.

$p$-region where the system follows the PTD. Moreover, the entropy rate for $L \leq m$ takes the value $H_{T} \simeq 1$, showing that for these word sizes the minority set is essentially random. Indeed, it follows from the MG rules that each step $i$ of the game can only be followed by one of the two possible outcomes (i.e. either a zero or a one); if the behavior is random, it turns out that $P_{i j}$ can only be equal to zero (if $i$ and $j$ can not be connected by the MG rules) or $1 / 2$. On the other hand the summation over the $\lambda$ values in Eq.2 will be equal to one; therefore it follows that $H_{T}=1$ for a random set built with the $\mathrm{MG}$ rules. Following the description of Manuca et.al [7], it can be said that one can not get information 'hidden' in $\left\{\mathcal{M}_{i}\right\}$ by using words of length $L \leq m$ : this will only be possible if one uses words one bit (or more) longer than $m$.

Figure 4 shows results for $m=5$ and 8 . It can be seen that they are similar to those described above for $m=2$, the main difference being that the value of $p^{*}$ gets smaller as $m$ increases. Notice that for $N=3721$, the case with $m=8$ has $z \simeq 0.07$, i.e. it still belongs to the first region.

\section{Conclusions}

We have studied a modification of the rules of the Minority Game, hoping to retain the main ideas of the model, and at the same time introducing a more realistic description of the agents. This was done through the introduction of certain number of interacting agents, that can know the decisions of their nearest neighbors, before they make their choice. As a result, the efficiency of the system improves if the fraction $p$ of $I$.A. is small, but it is worst when this fraction approaches $p \simeq 1$. The relation of the reduced variance $\mu_{r}$ with $p$, for $z<z_{c}$ resembles the relation of $\mu_{r}$ with $m$; in fact, for every value of $z$ there is an optimal value of $p, p^{*}(z)$, for which one has the minimum value of $\mu_{r}$. Thus, for a small fraction of $I . A$. the system remains in the PTD regime, sweeping through the whole set of histories; but when this fraction is greater 
than $p^{*}(z)$ (but still within the first, informationally efficient region), it falls into a cyclic, and in general non ergodic motion. In this sense, the use of the entropy rate proved to be a sensible tool to study the sequence of histories visited.

\section{References}

[1] D. Challet, Y.C. Zhang, Physica A 246 (1997) 407, and Physica A 256 (1998) 514.

[2] W.B. Arthur, Amer. Econ. Assoc. Papers and Proc. 84 (1994) 406.

[3] A collection of papers and preprints on this subject can be seen at the web site http://www.unifr.ch/econophysics/.

[4] M. Paczuski, K. Bassler, A. Corral, Phys. Rev. Lett. 84 (2000) 3185.

[5] T. Kalinowski, H-J Schulz, M. Briese, Physica A 277 (2000) 502.

[6] S. Moelbert, P. De Los Rios, Physica A 303 (2002) 217.

[7] R. Manuca, Y. Li, R. Riolo, R. Savit, Physica A 282 (2000) 559. A previous version of this paper was adap-org/9811005. See also R.Savit, R. Manuca, R. Riolo, adap-org/9712006.

[8] R. Savit, R. Manuca, R. Riolo, Phys. Rev. Lett. 82 (1999) 2203.

[9] D. Challet, M. Marsili, Phys. Rev. E 60 (1999) R6271. D. Challet, M. Marsili, R. Zecchina, Phys. Rev. Lett. 84 (2000) 1824.

[10] A. Cavagna, J. P. Garraham, I. Giardina, D. Sherrington, Phys. Rev. Lett. 83 (1999) 4429 .

[11] In fact, for other types of interactions this factor plays a rather relevant role (I. Caridi and H. Ceva, work in preparation).

[12] N.F. Johnson, M. Hart, P.M. Hui, Physica A 269 (1999) 1.

[13] I. Caridi, H. Ceva, Physica A 317 (2003) 247.

[14] T.M. Cover and J.A. Thomas, Elements of information theory, Chapter 4, Wiley series in telecommunications (1991).

[15] We have also studied a related set, by choosing words of length $L$ that are contiguous, but do not share any bit (one can form $M / L$ words of this kind). We found that the periodic behavior is also detected by this set of words, but its behavior for small values of $p$ is not very clear. 